\title{
Análisis comparativo lingüístico entre las versiones en inglés y español del Capítulo 17: Ambiental. TLC 1
}

\section{(Comparative Linguistic Analysis of the Englishand Spanish Versions of Chapter 17: Environment. CAFTA)}

\author{
Allan Pineda Rodríguez ${ }^{2}$ \\ Universidad Nacional, Costa Rica
}

\begin{abstract}
resumen
Partiendo de un texto, a manera de muestra, este análisis comparativo de las versiones en español e inglés desarrolla tres aspectos principales: la contextualización del documento (función, destinatario, género discursivo, etc.); los alcances de la traducción jurídica; y el examen de diez casos que presentan problemas traductológicos de interés para la disciplina. De todo lo expuesto, se extraen algunas recomendaciones prácticas.
\end{abstract}

\begin{abstract}
Using a text chosen as a point of departure, this comparative analysis of the Spanish and English versions addresses three main aspects: the contextualization of the document (function, target audience, genre of discourse, etc.); the scope of legal translation; and the analysis of ten cases of particular interest within the discipline of translation studies. With this basis, practical recommendations are given.
\end{abstract}

1 Recibido: 24 de mayo de 2014; aceptado: 7 de agosto de 2014.

2 Escuela de Literatura y Ciencias del Lenguaje. Correo electrónico: allan.pineda.rodriguez@una.cr

$$
L_{\text {etras }} 56 \text { (2014), ISSN 1409-424X; EISSN 2215-4094 } 123
$$


Palabras clave: traducción jurídica, tratados comerciales e internacionales, análisis lingüístico comparativo

Keywords: legal translation, trade agreements, international treaties, comparative linguistic analysis

\section{Introducción}

Latraducción jurídica, en particular la detratadosinternacionales constituye un desafío para el traductor, no sólo en lo que se refiere al vocabulario técnico legal y de otras disciplinas, sino también a las diferencias entre idiomas, culturas, y si se toman en cuenta los diferentes sistemas legales de las partes. Desde finales del siglo xx surgieron en Centroamérica movimientos a favor y en contra de tratados de libre comercio (TLC) entre República Dominicana, Centroamérica y Estados Unidos. Parte de esa discusión se centra en las ventajas o desventajas que podrían traer para las partes. Sin embargo, de igual forma hay que reconocer que las interpretaciones que se puedan dar al texto del tratado — en los idiomas en que se redacte - tienden a inclinar la balanza. De ahí lo delicado y cuidadoso que debería ser el manejo lingüístico de dichos documentos.

Puesto que no se ha podido establecer si existe un documento original de este TLC y su respectiva traducción o si fue una redacción conjunta, queremos en primer lugar dejar claro que para efectos de este artículo lo que se pretende hacer es un análisis comparativo lingüístico como si se tratara de la traducción de un texto del inglés al español. A pesar de ese riesgo, ya que no hay seguridad de que sean original y traducción, esta investigación parte de ese supuesto.

Este artículo no tiene el propósito de aclarar las dudas que han surgido en las versiones del documento ni tampoco toma parte a favor o en contra de las interpretaciones. Se intenta desvelar algunos problemas de traducción o redacción y de anisomorfismo, que pueden surgir cuando se trabaja con este tipo de documentos. Se ha seleccionado el 
capítulo de «Ambiente» del Tratado de Libre Comercio entre República Dominicana, Centroamérica y Estados Unidos.

ElCapítulo 17, «Ambiental», fue seleccionado como muestra de los veintidós capítulos que contiene el tratado. La razón fundamental de elegir esa sección para el análisis nace del interés de tener un texto que fuese neutral y de conocimiento común; es decir, que no implicase tantos aspectos jurídicos, comerciales, financieros o políticos y nos permitiese centrarnos en los aspectos lingüísticos del texto. Por otro lado, al tenerse Costa Rica como una nación con una trayectoria ambiental bastante reconocida internacionalmente, sería interesante relacionar el contenido de este artículo con el trasfondo ambiental y el nombre del país.

La primera parte de este estudio hace una breve descripción del documento con el que se trabajó y su respectivo análisis textual. En la segunda parte mencionamos algunos aspectos fundamentales de la traducción jurídica de tratados a nivel internacional. En la tercera parte se presentan los casos y problemas con su respectivo análisis y al final se ofrecen las conclusiones correspondientes.

\section{El Tratado de Libre Comercio}

El Tratado de Libre Comercio entre República Dominicana, Centroamérica y Estados Unidos es un acuerdo que busca beneficios económicos y comerciales entre estos países. Las negociaciones para su elaboración se iniciaron en 2003 y culminaron en enero de 2004, y desde entonces hasta 2009 cada uno de los países participantes ha empezado a ratificarlo y a ponerlo en práctica. El Ministerio de Comercio Exterior de Costa Rica lo define como:

Un instrumento para fomentar la producción, la inversión nacional y extranjera, el empleo y el comercio; en la medida en que crea mejores condiciones para realizar nuestro comercio con Estados Unidos que las que tenemos hoy. Es decir, es un 
instrumento de la política económica, que busca crear más opciones de crecimiento económico ${ }^{3}$.

El tratado consta de veintidós capítulos; además, contiene tres anexos. El capítulo 17, bajo el título «Ambiental», se toma como base para este estudio ${ }^{4}$. Este capítulo está formado por doce artículos y aborda algunos temas como la protección ambiental, la aplicación y observancia de la legislación ambiental, la participación pública, la cooperación, los mecanismos de consulta, la relación con acuerdos ambientales, etc.

Antes de proceder con el análisis textual es necesario indicar que tanto la versión en inglés como la del español se dan como auténticas, tomadas del sitio web del Ministerio de Comercio Exterior de Costa Rica ${ }^{5}$. El capítulo 22 del tratado, que se refiere a las «Disposiciones finales», estableceensuArtículo 22.9 que «los textos eninglés yespañol de este Tratado son igualmente auténticos». Ambas versiones tienen igual validez, dependiendo del idioma oficial de cada país. Ninguna parte del tratado, ni otros documentos informativos oficiales afines, mencionan la traducción del documento y lo único que establecen es lo que indica el folleto titulado Lo más importante sobre el TLC del Ministerio de Comercio Exterior de Costa Rica:

De febrero a mayo del 2005, los seis países trabajaron en la revisión jurídica y el cotejo lingüístico del texto negociado, lo que significó que un grupo de abogados de todos los países revisó la

3 Costa Rica, Ministerio de Comercio Exterior. (2006). Lo más importante sobre el TLC., p. 3. Recuperado el 10 de enero de 2014, de <http://www.comex.go.cr/archivo/cafta/Lo\%20mas\%20 importante\%20sobre\%20el\%20TLC.pdf>.

4 En español (Costa Rica): Ministerio de Comercio Exterior (2004), Capítulo 17: Ambiental. Tratado de Libre Comercio República Dominicana-Centroamérica-Estados Unidos. Recuperado el

10 de enero de 2014, de <http://www.comex.go.cr/tratados/vigentes/cafta/texto\%20tratado/ capitulo\%2017/00_capitulo17_cafta.pdf>. En inglés (Estados Unidos de América): Office of the United States Trade Representative. (2004). Chapter 17: Environment. The Dominican RepublicCentral America-United States Free Trade Agreement. Recuperado el 10 de enero de 2014, de <http://www.ustr.gov/sites/default/files/uploads/agreements/cafta/asset_upload_file9_3937.pdf>.

5 <http://www.comex.go.cr> (2004: 22-2). 
coherencia entre los capítulos y que las versiones en español e inglés fueran equivalentes ${ }^{6}$.

Según el Tratado de Libre Comercio República DominicanaCentroamérica-Estados Unidos: documento explicativo, durante las negociaciones las partes se reunieron en diversos momentos y lugares para debatir los diferentes temas. En el documento explicativo no se indica en qué idioma se llevaron a cabo las negociaciones, no se menciona el uso de intérpretes, tampoco se indica si existió un documento original y un documento traducido. Sin embargo, en otro documento consultado titulado El proceso de negociación de un tratado de libre comercio con Estados Unidos: la experiencia del tratado de libre comercio entre Centroamérica, Estados Unidos y República Dominicana ${ }^{7}$, su autora hace referencia a que como parte del proceso de coordinación centroamericana, la Secretaría de Integración Económica Centroamericana (SIECA) se haría cargo de contratar la traducción e interpretación. Este es el único indicio de que hubo una etapa de traducción. No obstante, tampoco se señala la dirección de la traducción, ni de si existió un documento o alguna sección del documento cuyo original fuese o el inglés o el español. Se deduce, por lo indicado en dicho documento, que efectivamente el origen de los documentos durante la negociación fue el idioma inglés y que una vez recibidos, negociados y discutidos se procedió a su redacción en español. Como parte de las conclusiones se intenta esclarecer si fue esa la dirección de la creación y redacción de las versiones, es decir, que en consecuencia existió un texto original en inglés y su contraparte traducida al español.

6 <http://www.comex.go.cr/archivo/cafta/Lo\%20mas\%20importante\%20sobre\%20el\%20TLC.pdf $>$ (2006) 23.

7 Anabel González, El proceso de negociación de un tratado de libre comercio con Estados Unidos: la experiencia del tratado de libre comercio entre Centroamérica, Estados Unidos y República Dominicana (Buenos Aires: BID-INTAL, 2006). 


\section{Análisis textual}

Conforme a los procedimientos de Christiane Nord sobre el análisis del texto fuente, se definirá la función o intención comunicativa del texto, el o los destinatarios, el tiempo y lugar de la recepción del texto, el medio de transmisión y el motivo para su producción o recepción ${ }^{8}$.

En primer lugar, como capítulo de un tratado comercial internacional, se trata de un texto administrativo; visto desde la perspectiva legislativa estaríamos hablando de un texto jurídico. Sin embargo, estos tipos textuales tienen principalmente la finalidad de informar, por lo que hablaríamos de un escrito cuya función primordial es la informativa. Siendo este un documento jurídico, habría que tomarlo como texto de aplicación del derecho, como lo es un contrato, pero en este caso las partes no son personas sino países negociantes. En cuanto a los destinatarios cabe mencionar a los respectivos gobiernos de cada país que forman parte del tratado y a sus correspondientes congresos nacionales, que en primera instancia son los encargados de su ratificación. También se mencionaría como otros destinatarios a todos los ciudadanos de los países participantes, en especial los interesados en desarrollar las relaciones comerciales entre las partes. En cuanto al tiempo, para todas las partes es el mismo, ya que todas las naciones han estado en el proceso desde su inicio. Ambas versiones salen a la luz pública de forma simultánea en los respectivos países. Con relación al ámbito de aplicación, se establece que el texto en inglés es para Estados Unidos y el texto en español es para el resto de países de Centroamérica y para República Dominicana. En cuanto al medio de transmisión, se trata de acuerdo legal, que lo clasifica dentro del género jurídico escrito y como tal posee las características propias de un acuerdo entre partes, redactado en un estilo formal, con la inclusión de sus capítulos, secciones y artículos como corresponde. Es

8 Christiane Nord, Text Analysis in Translation (Amsterdam: Rodopi, 1991). 
un texto que se encuentra a disposición de los ciudadanos a través de diversas instituciones gubernamentales y se ha divulgado por medio de sus respectivas plataformas en internet. No es un texto bilingüe y es importante recalcar que existen dos versiones del mismo escrito; cada documento es independiente y le corresponde a cada gobierno hacerse cargo de su difusión y explicación, pues al ser un documento jurídico es necesario aclarar a sus ciudadanos las dudas que puedan surgir luego de su lectura. Para finalizar se establece que el motivo de su creación es la de implantar las reglas del juego, o sea los reglamentos y normas, para negociar de manera clara y abierta entre los países involucrados en el tratado.

\section{Traducción jurídica y tratados}

Se trabaja con dos textos, una versión en inglés y otra en español. Como no es posible tener certeza de que existe un texto original y aunque se considere al texto inglés como original y al español como texto meta, puede que algunas de las teorías y libros que hacen referencia a la traducción jurídica del inglés al español no se apliquen del todo al presente trabajo; sin embargo, las nociones básicas de esta práctica pueden ser de gran utilidad, por lo menos para establecer generalidades que se utilizan en la redacción y traducción de este tipo de documentos.

El lenguaje jurídico se sitúa dentro del ámbito de los lenguajes especializados. La traducción de textos procedentes de un campo de especialidad se engloba como traducción especializada. Además, los textos legales contienen características específicas delámbito jurídico, como por ejemplo el uso de vocabulario especializado. Si bien no se trata de documentación jurídica de tipo de «acción civil» o «causa criminal», ni se consideran a jueces, ni tribunales, se trata de un documento legal que tendrá esa validez bajo las respectivas legislaciones y los países correspondientes que forman parte del tratado, aunque esté redactado en varios idiomas. 
En lo que corresponde a la redacción de documentación legal en diversos idiomas, Anabel Borja se refiere a la normalización de la traducción de textos jurídicos cuando se está ante textos multilingües ${ }^{9}$. Señala una tendencia a la normalización de documentos, esencialmente de los de carácter informativo y referencial. También establece que tal tendencia se aplica a todo tipo de documentos, en especial a los textos diplomáticos, técnicos y comerciales y entre ellos está la documentación jurídica. Para Borja, este tipo de documentos comparten el hecho de ser parte fundamental en las transacciones internacionales, y por obvias razones es muy común que se encuentren traducidos en diversos idiomas ${ }^{10}$.

Susan Šarčević da mejor información sobre la traducción de este tipo de documentos. En New Approach to Legal Translation dedica un capítulo a la traducción de instrumentos multilaterales ${ }^{11}$. Algunos aspectos que destaca, además de la estructura de los tratados, son el multilingüismo, la interpretación yel principio de igual autenticidad, la traducción y la naturaleza especial de los tratados, etc. A continuación se presentan algunos elementos de importancia para la traducción de este tipo de textos.

Respecto a la estructura de los tratados, Šarčević señala que este tipo de documentos se pueden dividir en título, preámbulo, parte principal y cláusulas finales ${ }^{12}$. Más adelante señala «...it suffices to note that these provisions [refiriéndose a las cláusulas que conforman la parte principal del tratado] are generally formulated in the same way as legal rules of municipal legislation $\gg^{13}$. Basta con reconocer que tanto para la redacción como para la traducción de los tratados es conveniente emplear textos que sirvan como referencia y que se

$9 \quad$ Anabel Borja Albi, El texto jurídico inglés y su traducción al español (Barcelona: Ariel, 2000) 171-172.

10 Borja, 172.

11 Susan Šarčević, New Approach to Legal Translation (La Haya: Kluwer Law International, 1997).

12 Šarčević, 131.

13 Šarčević, 132. 
adopten como textos normativos para la creación de este tipo de documentos legales.

En cuanto a la interpretación de los tratados Šarčević llama la atención en el sentido que la más leve discordancia entre textos con un mismo contenido y propósito, pero redactados en más de un idioma, puede acarrear situaciones o incidentes políticos de importancia que podrían poner en peligro las relaciones entre los países o las partes ${ }^{14}$. De igual forma aclara: «The demand for flawless decision-making automatically places additional pressure on translators, forcing them to carefully weigh each and every word and give special consideration to all possible interpretations and misinterpretations when formulating the text ${ }^{15}$.

Los traductores de este tipo de documentostienenque ser cautelosos para redactaresos instrumentos legales. Denuevo Šarčević advierte que no es recomendable que los traductores utilicen su creatividad con el idioma, y que más bien se deben apegar lo más que puedan al texto original ${ }^{16}$. Como reto la autora advierte que «...translators of treaties should strive to produce an idiomatic text that reads like an original, yet preserves sufficient interlingual concordance so as to prevent ambiguity and possible misinterpretations» ${ }^{17}$. Otro aspecto significativo es que los traductores no deben asumir la responsabilidad de aclarar aquellas situaciones confusas, oscuras o ambiguas ya que pueden dar al traste con las negociaciones y malinterpretar las intenciones de las partes ${ }^{18}$.

Conviene también hacer detenerse en la autenticidad de los tratados que se redactan en más de un idioma. Para ello se retoma lo que establece la Vienna Convention on the Law of Treaties between States and International Organizationsorbetween International Organizations. Este documento fue redactado por la Comisión de Derecho Internacional de las Naciones Unidas y su alcance es únicamente para

\footnotetext{
14 Šarčević, 200.

15 Šarčević, 201.

16 Šarčević, 202.

17 Šarčević, 203.

18 Šarčević, 204.
} 
tratados por escrito entre los Estados. La Convención establece en su artículo 33, «Interpretation of treaties authenticated in two or more languages», lo siguiente:

1. When a treaty has been authenticated in two or more languages, the text is equally authoritative in each language, unless the treaty provides or the parties agree that, in case of divergence, a particular text shall prevail.

2. A version of the treaty in a language other than one of those in which the text was authenticated shall be considered an authentic text only if the treaty so provides or the parties so agree.

3. The terms of a treaty are presumed to have the same meaning in each authentic text.

4. Except where a particular text prevails in accordance with paragraph 1, when a comparison of the authentic texts discloses a difference of meaning which the application of articles 31 [General rule of interpretation] and 32 [Supplementary means of interpretation] does not remove, the meaning which best reconciles the texts, having regard to the object and purpose of the treaty, shall be adopted ${ }^{19}$.

Considerado lo anterior, los tratados redactados en más de un idioma se toman como auténticos y tendrán la misma validez siempre y cuando así se establezca en el mismo tratado. Por ello la necesidad y el interés de que las traducciones realmente se realicen con la mayor atención posible, ya que no se trata solo de versiones, sino de textos legales y auténticos que tendrán la validez correspondiente entre las naciones y ante todas las instancias jurídicas que así lo requieran.

19 Naciones Unidas. Codification Division, Office of Legal Affairs (1986). Vienna Convention on the Law of Treaties between States and International Organizations or between International Organizations. (1980) Recuperado el 15 de febrero de 2014, de <http://legal.un.org/ilc/texts/ instruments/english/conventions/1_2_1986.pdf>, p. 17. 


\section{Análisis lingüístico de casos representativos}

A continuación se muestran diez casos de comparaciones lingüísticas entre las que hay problemas de traducción o redacción y de anisomorfismo de las versiones en inglés y en español del capítulo analizado. Los primeros ocho casos (Títulos, Omisiones e inconsistencia, Incoherencia gramatical, Definiciones, Uso de siglas, y las palabras «submission», «secretariat» $\mathrm{y}$ «monitoring») se seleccionan para mostrar situaciones lingüísticas que crean alguna duda en cuanto a la redacción o traducción realizada, ya sea por forma o por fondo. Por el contrario, los últimos dos casos (Expresiones latinas y la palabra «domestic») destacan aciertos en su «traducción», pero al mismo tiempo ejemplifican que entre estos textos bilingües legales existe esa situación de anisomorfismo de la cual todos los traductores deben ser conscientes.

\section{Caso 1: Títulos}

Versión en inglés:

- Article 17.4: Voluntary Mechanisms to Enhance Environmental Performance

Versión en español:

- $\quad$ Artículo 17.4: Mecanismos Voluntarios para Mejorar el Desempeño Ambiental

\section{Comentario:}

Aquí se nota que en la versión en inglés los títulos incluyen el uso de mayúsculas, excepto las preposiciones y artículos. En la versión en español podemos notar que se sigue el mismo patrón que la versión inglesa al emplear las mayúsculas del mismo modo. Esto sucede con todos los títulos de los artículos. De esta forma, existe la posibilidad de pensar que la versión original sea la inglesa. 


\section{Caso 2: Omisión e inconsistencia}

Versión en inglés:

- $\quad$...the ECA, taking into account guidance regarding those goals provided by the Council and the Environmental Cooperation Commission established under the ECA...

- The Parties also recognize the continuing importance of current and future environmental cooperation activities in other fora.

- exchanging information and experiences between Parties wishing to perform environmental reviews, including reviews of trade agreements, at the national level; and...

Versión en español:

- $\quad . . y$ del ACA, tomando en consideración los lineamientos en relación con dichas metas dispuestas por el Consejo y la Comisión de Cooperación establecida en el ACA...

- Las Partes además reconocen la continua importancia de las actuales y futuras actividades de cooperación en otros foros.

- intercambio de información y experiencias entre las Partes que deseen llevar a cabo revisiones ambientales, incluyendo revisiones de los tratados de libre comercio, a nivel nacional; y...

\section{Comentario:}

En el primer caso se observa la omisión de environmental: EnvironmentalCooperation Commissionpor «Comisión de Cooperación»; y future environmental cooperation activities por «futuras actividades de cooperación». Si bien es cierto no es tan grave el hecho que se omita la palabra correspondiente «ambiental», podría ser necesario incluirla, pues se trata del capítulo que hace referencia precisamente al tema del ambiente. Sería conveniente agregar la palabra en cuestión para aclarar que se trata de una comisión de cooperación que trabaja precisamente en esa área, en el ambiente; y en el segundo ejemplo 
es recomendable aclarar el tipo de actividades de cooperación que se piensa realizar en el futuro.

El tercer ejemplo es con trade agreements por «tratados de libre comercio». Es de destacar que en esta ocasión lo que sucede, por el contrario, es que se agrega la palabra «libre» en la versión en español. Este cambio puede llevar a una confusión en las versiones pues no es lo mismo un «tratado de comercio» y un «tratado de libre comercio». Por lo tanto existe inconsistencia en las versiones.

\section{Caso 3: Incongruencia gramatical (estructura paralela en un listado)}

Versión en inglés

- $\quad$ may consider any relevant technical, scientific, or other information:
(a) that is publicly available;
(b) submitted by interested persons;
(c) submitted by national advisory or consultative committees;
(d) developed by independent experts; or
(e) developed under the ECA.

Versión en español:

- $\quad$ podrá tomar en cuenta toda información pertinente, de naturaleza técnica, científica o de otra índole que:
(a) esté disponible al público;
(b) sea presentada por personas interesadas;
(c) sea presentada por comités nacionales consultivos o asesores;
(d) elaborada por expertos independientes; o
(e) desarrollada bajo el ACA.

\section{Comentario:}

En este ejemplo hay incoherencia estructural en la redacción de esta lista. El problema se presenta por el uso de participios en los puntos d) y e) de la versión en español. En la del inglés después del 
sustantivo information se utiliza en esos puntos el participio developed. Mientras tanto en la versión en español si luego de la conjunción «que» en los puntos a), b) y c) se utilizó el verbo «ser»o «estar» en su conjugación de presente subjuntivo (esté y sea), no se continuó con esos verbos en los puntos d) y e) y se empleó las formas de participio, que son «elaborada» $\mathrm{y}$ «desarrollada». Por lo tanto el texto en español es incoherente en las siguientes construcciones gramaticales en el punto d) «información (...) o de otra índole que elaborada por...»y también en el punto e) «información (...) o de otra índole que desarrollada bajo...». Nuevamente, en apariencia, se está haciendo una traducción del inglés al español y en este preciso ejemplo se hace una copia de la estructura inicial (en inglés) de esos dos puntos y también se emplea el participio de los verbos (en español).

\section{Caso 4: Definiciones}

Versión en inglés:

- $\quad$ environmental law means any statute or regulation of a Party...

- $\quad$ For purposes of Article 17.7.5, judicial or administrative proceeding means:

Versión en español:

- legislación ambiental significa cualquier ley o regulación de una Parte...

- $\quad$ Para los efectos del Artículo 17.7.5, «procedimiento judicial o administrativo» significa:

\section{Comentario:}

Existe incoherencia por el uso en el formato del texto, específicamente con el estilo de la fuente (o letra) utilizado «negrita» y de las comillas. En la versión en inglés se emplea la negrita para resaltar aquella palabra o palabras de las cuales se establecerá su definición. En la versión en español se combina el uso de la negrita y el uso de las 
comillas, lo cual podría llegar a confundir además de que no muestra un patrón de consistencia en un tratado de esta índole. Es de resaltar que en otros tratados costarricenses en español se ha empleado tanto la negrita como el uso de las comillas para aclarar las definiciones, pero se ha mantenido una sola de las opciones, y no como en estos ejemplos donde se han mezclado.

\section{Caso 5: Uso de siglas}

Versión en inglés:

- ...may file a submission under that agreement with the Secretariat of the NAAEC Commission for Environmental Cooperation asserting that the United States is failing to effectively enforce its environmental laws.

Versión en español:

- ...puede presentar una comunicación bajo ese acuerdo al Secretariado delACAAN Comisión para CooperaciónAmbiental que asevere que los Estados Unidos está incumpliendo en la aplicación efectiva de su legislación ambiental.

\section{Comentario:}

La frase en español de «Secretariado del ACAAN Comisión para Cooperación Ambiental» es un rotundo ejemplo de una mala interpretación en el uso y colocación de siglas. La versión en inglés por su parte es muy clara Secretariat of the NAAEC Commission for Environmental Cooperation. Puede notarse que aunque se empleó el correspondiente equivalente tanto para las siglas NAAEC (NorthAmerican Agreement on Environmental Cooperation) por «ACAAN» (Acuerdo de Cooperación Ambiental de América del Norte), como para la traducción de Commission for Environmental Cooperation por «Comisión para Cooperación Ambiental» lo importante es señalar que la ubicación de la sigla no corresponde a la estructura gramatical que debería tener la 
versión en español; la versión correcta hubiese sido «Secretariado de la Comisión para Cooperación Ambiental del ACAAN». De nuevo es notorio con este ejemplo que existe un documento original en inglés y una traducción al español, ya que en este caso se está utilizando la estructura gramatical del inglés en ambas versiones.

\section{Caso 6: «submission»}

Versión en inglés:

- Any person of a Party may file a submission asserting that a Party is failing to effectively enforce its environmental laws.

- the submission is drawn exclusively from mass media reports. Where the secretariat makes such a request, it shall forward to the Party a copy of the submission and any supporting information provided with the submission.

Versión en español:

- Cualquier persona de una Parte podrá remitir comunicaciones que aseveren que una Parte está incumpliendo en la aplicación efectiva de su legislación ambiental.

- $\quad$ si la petición se basa exclusivamente en noticias de los medios de comunicación. Cuando el secretariado solicite una respuesta, remitirá a la Parte una copia de la comunicación, así como cualquier otra información de apoyo que la acompañe.

\section{Comentario:}

Para Šarčević, cuando hay terminología especializada, una vez seleccionado un término equivalente, debe mantenerse y evitar el uso de sinónimos, ya que puede pensarse que se hace referencia a otro concepto diferente ${ }^{20}$. En esta situación se emplea el término «comunicación» como el equivalente de submission; sin embargo, su uso no 
es constante y más adelante se cambia por «petición», lo cual podría crear alguna confusión de si realmente se trata de la «comunicación» o si se trata de alguna «petición» especial o diferente.

\section{Caso 7: «secretariat»}

Versión en inglés:

- $\quad$.... Such submissions shall be filed with a secretariat or other appropriate body («secretariat») that the Parties designate.

- The secretariat may consider a submission under this Article if the secretariat finds that the submission:

Versión en español:

- Dichas comunicaciones serán dirigidas a una secretaría u otro organismo apropiado («secretariado»), que las Partes designen.

- El secretariado podrá considerar una comunicación bajo este Artículo, si el secretariado encuentra que:

\section{Comentario:}

Se utiliza el equivalente en español de «secretaría» y «secretariado» por el término secretariat en inglés. No queda clara la razón por la que se emplean los dos términos en español. En la versión inglesa se utiliza el término una vez en el párrafo y luego se escribe entre comillas y paréntesis, lo cual da la idea de que es así como se referirá a este organismo en el resto del texto. Su contraparte en español hace lo mismo. Sin embargo, para estas situaciones en los textos jurídicos de este tipo en español lo que se utiliza es la estructura «en lo sucesivo...» o «de ahora en adelante...» y se escribe el término tal y como se utilizará por el resto del texto. Este es otro de los ejemplos donde se observa un patrón idéntico en la estructura de ambas versiones y que da la idea de que se está en presencia de una traducción del inglés al español. 


\section{Caso 8: «monitoring»}

Versión en inglés:

- ...or reduce environmental impacts, environmental monitoring, and collection of baseline data...

- ...of the Party's mechanisms for monitoring its environmental enforcement.

- Versión en español:

- ...o reducir los impactos ambientales, monitoreo ambiental y la recolección de datos para establecer líneas base...

- $\quad$...de los mecanismos de la Parte referentes al monitoreo de la aplicación de la legislación ambiental.

\section{Comentario:}

El diccionario de la RAE no incluye la voz «monitorear»; cuando mucho, se encuentra la palabra «monitor» en su acepción de individuo como «persona que guía el aprendizaje deportivo, cultural, etc.», o en su acepción de objeto como «aparato receptor que toma las imágenes directamente de las instalaciones filmadoras y sirve para controlar la transmisión», entre otras muchas acepciones; o «monitorizar» como verbo relacionado a «observar mediante aparatos especiales el curso de uno o varios parámetros fisiológicos o de otra naturaleza para detectar posibles anomalías» ${ }^{21}$. En este caso se puede notar cómo se utiliza el término «monitorear» para describir la acción de seguimiento y control que debe hacerse en el ámbito ambiental. En Alcaraz Varó y Hughes se menciona el uso de préstamos y calcos cuando se refiere al inglés jurídico y su traducción al español, «Cuando dos culturas han estado en contacto, aunque haya sido por un período corto de tiempo,

21 Real Academia Española (RAE), «Monitor», Diccionario de la lengua española (22 ${ }^{\mathrm{a}}$ ed., 2001). Consultado el 15 de noviembre de 2014, <http://lema.rae.es/drae/?val=monitor >; RAE, «Monitor», Diccionario de la lengua española (avance, $23^{\mathrm{a}}$ ed., 2014). Consultado el 15 de noviembre de 2014, <http://lema.rae.es/drae/srv/search?id=RsO0Y5fvx2x5rI3bCqI>; RAE, «Monitorizar», Diccionario de la lengua española (22a ed., 2001). Consultado el 15 de noviembre de 2014, <http://lema.rae.es/drae/?val=monitorizar>. 
normalmentese hanenriquecido conceptualyexpresivamente, prestándose palabras y expresiones entre sí» ${ }^{22}$. Además, se analiza el ejemplo del inglés implement, que se traduce por «implementar» habiendo otras opciones en español, como «ejecutar» o «realizar» ${ }^{23}$. Siendo el ejemplo de «monitoring» un caso similar, se podría establecer que el idioma español, específicamente elque se habla en Centroamérica, está influido por rasgos culturales de los Estados Unidos, y más al referirse a un tratado entre esa nación y el resto de países centroamericanos.

Salta a la vista la posibilidad de que los textos del tratado sean el original en inglés y su traducción en español. Es esencial considerar la influencia del idioma inglés en esta área del continente americano. Sin embargo, se ha encontrado en otros tratados centroamericanos tanto el uso del término «monitorear», como la utilización de la palabra «vigilar». Por tanto, si bien existe la tendencia a copiar el término inglés y adaptarlo al español, también existe la posibilidad de buscar alternativas para que el documento español se lea de una forma más natural. Además es importante reconocer que esa influencia del inglés está presente no solo en Centroamérica sino que incluso seencuentra en organizaciones internacionales como la Comisión para la Cooperación Ambiental de América del Norte que utiliza el vocablo «monitorear» en sus artículos de difusión, como en este ejemplo: «Monitoreo y Evaluación de los Contaminantes en América del Norte» ${ }^{24}$.

22 V. E. Alcaraz Varó y B. Hughes, Legal Translation Explained (Manchester, R. U. : St. Jerome, 2002) 88.

23 Alcaraz Varó y Hughes, 90.

24 El título en inglés de este artículo es: Monitoring and Assessing Pollutants across North America. En inglés: Commission for Environmental Cooperation (2010) Project 9 - Monitoring and Assessing Pollutants across North America. Documento original. Recuperado el 15 de febrero de 2014, de <http://www.cec.org/Storage/86/8192_OP10-Proj9-Monitoring_Pollutants_EMA_SMOC_WG18Dec09-Draft3-e2-final.pdf>; en español: Comisión para la Cooperación Ambiental (2010) Proyecto 9-Monitoreo y Evaluación de los Contaminantes en América del Norte. Traducción. Recuperado el 15 de febrero de 2014, de <http://www.cec.org/Storage/93/9045_OP10-Proj9Monitoring_Pollutants_EMA_SMOC_WG-18Dec09-Draft3-e2-final-s1.pdf > . 


\section{Caso 9: Expresiones latinas}

Versión en inglés:

- Accordingly, the Parties understand that a Party is in compliance with subparagraph (a) where a course of action or inaction reflects a reasonable exercise of such discretion, or results from a bona fide decision regarding the allocation of resources.

Versión en español:

- En consecuencia, las Partes entienden que una Parte está cumpliendo con el subpárrafo (a) cuando un curso de acción o inacción refleje un ejercicio razonable de tal discrecionalidad, o derive de una decisión adoptada de buena fe respecto de la asignación de recursos.

\section{Comentario:}

En este caso no estamos ante un uso impropio de expresiones latinas. Por el contrario, el trato que se le da tanto a la expresión latina en inglés como a su no utilización en la versión española es lo aconsejable, según expertos. Alcaraz Varó y Hughes explican que el inglés jurídico ha sido influenciado en algún sentido por el derecho romano y como tal se ha adoptado el uso de ciertos latinismos, es uno de sus rasgos principales. En cuanto a su traducción, los autores señalan que «The decision as to whether to translate or not must be made in accordance with standard practice among the members of the legal community in the target-language system ${ }^{25}$. Siempre bajo el mismo concepto del uso de los latinismos, Šarčević agrega que el hecho que los juristas (ingleses) empleen frases latinas hace más difícil el trabajo de los traductores ${ }^{26}$. Por lo anterior en este caso el traducir la frase bona fide por «de buena fe» y no dejarla en latín es lo ideal en los textos en español.

25 Alcaraz Varó y Hughes, 5.

26 Šarčević, 264. 


\section{Caso 10: «domestic»}

Versión en inglés:

- Recognizing the right of each Party to establish its own levels of domestic environmental protection and environmental development policies and priorities...

- The Parties recognize that it is inappropriate to encourage trade or investment by weakening or reducing the protections afforded in domestic environmental laws.

- ...shall review and respond to them in accordance with its domestic procedures.

- $\quad$...in protecting the environment globally and domestically and that their respective implementation of...

- a domestic judicial, quasi-judicial, or administrative action pursued by the Party in a timely fashion and in accordance with its law.

- exchanging information on domestic implementation of multilateral environmental agreements...

Versión en español:

- Reconociendo el derecho de cada Parte de establecer sus propios niveles de protección ambiental y sus políticas y prioridades de desarrollo ambiental...

- Las Partes reconocen que es inapropiado promover el comercio o la inversión mediante el debilitamiento o reducción de las protecciones contempladas en su legislación ambiental interna.

- ...las revisará y responderá de acuerdo con sus procedimientos internos.

- ...en la protección del ambiente a nivel global y nacional, y que la importancia de la implementación respectiva de...

- una actuación judicial, cuasijudicial o administrativa realizada por una Parte de manera oportuna y conforme a su legislación.

- $\quad$ intercambio de información sobre la implementación a nivel nacional de acuerdos ambientales multilaterales... 


\section{Comentario:}

Al igual que el caso anterior de las expresiones latinas, encontramos un uso de equivalencias entre el término domestic en inglés y su contraparte en español. Este ejemplo demuestra que, aunque hay errores o dudas en los casos del 1 al 8, también hay aciertos en el trato de algunos términos que podrían provocar duda al equipararse entre ambos idiomas. El término «doméstico» en español se refiere a aquello relacionado a la casa: «tareas domésticas», «animales domésticos», etc. Si en español tomamos la palabra «doméstico» con el mismo sentido del inglés entonces se estaría utilizando un «falso amigo» ya que en inglés además del significado en español (relacionado a la casa) también se utiliza para todo aquello relacionado a un país hacia lo interno: «domestic trade », «domestic goods», etc.

Para evitar el uso de la palabra «doméstico» se han empleado en este texto otros recursos como el uso del adjetivo posesivo ( $\mathrm{su}$, sus) para referirse a situaciones internas del país o precisamente la utilización de otras palabras como «nacional», «a nivel nacional» $\mathrm{o}$ el mismo adjetivo «interno».

\section{Conclusiones}

Si en un principio se tenía dudas con respecto a si realmente se trataba de una redacción conjunta de ambas versiones, ahora es posible tener una mayor certeza de que el supuesto de original inglés y traducción español es cierto, no solo porque hubo un equipo de traducción e interpretación en el proceso, sino que al tomar como ejemplo los casos 1, 4, 6, 7 y 8 se muestra que la versión en inglés se utilizó como referente y base para la versión en español. Existe un marcado uso de patrones gramaticales, semánticos y de estilo que se utilizan en la versión en español del tratado y que no deberían ser comunes en este idioma. Si a esto se suma el carácter históricamente dominante de la cultura y economía de los Estados Unidos sobre Latinoamérica, y en particular sobre Centroamérica, se concluye que 
aunque las negociaciones se dieron en ambos idiomas, es probable que la versión final haya sido en inglés y que una vez estudiado y analizado se haya traducido al español.

Al comparar los ejemplos con otros tratados costarricenses, elaborados originalmente en español, podrían establecer algunos criterios de redacción que deberían seguirse en los tratados que sean traducidos al español (o versiones en español) para que tengan la naturalidad que el idioma requiere y de la cual carece en momentos el documento en español analizado. Entre los aspectos más relevantes en su estructura podemos señalar los siguientes:

- Capítulos con número arábigo (o romano) en lugar de palabras;

- Uso de mayúsculas en el nombre del capítulo;

- Artículos con la siguiente numeración:

Artículo 8.01

Artículo 1-04

- Mayúsculas solo al inicio del nombre del artículo o cuando algún sustantivo especial lo requiera.

Es probable que si se examinara el resto de capítulos se podrían encontrar errores o situaciones similares a las aquí descritas. Este análisis lingüístico comparativo nace del interés por los idiomas y los procesos de traducción; no hay intención de examinar, desde el punto de vista jurídico o comercial, los alcances o las intenciones del documento. Si se deseara hilar más fino y ceñirse al carácter legal del tratado, se podrían descubrir problemas de ambigüedad jurídica, que en definitiva deberían tratarse con extremo cuidado. Esto permite concluir que la traducción en ámbito jurídico es un verdadero desafío; para efectuar un trabajo de traducción en este campo hay que prepararse muy bien y además tener suficiente conocimiento jurídico y lingüístico para realizar una tarea acorde con lo requerido y lo que se espera de un traductor competente. 